\title{
A Multiplex Quantitative Polymerase Chain Reaction Using Applied Biosystems 7500 Fast System for Simultaneous Identification of Three Campylobacter Species with Potential Applications to Food Analysis
}

\author{
Kun C. Liu
}

\begin{abstract}
Consumption of Campylobacter-contaminated food is one of the most common causes of bacterial diarrhea. A previously developed quantitative polymerase chain reaction (qPCR) utilizing the SmartCycler instrument platform for identification of Campylobacter jejuni, Campylobacter coli, and Campylobacter lari had to be modified to address the recent discontinuation of the SmartCycler system. In this study, a multiplex qPCR assay was optimized on the Applied Biosystems 7500 Fast (AB7500F) platform to continue using qPCR for the identification of three target Campylobacter spp. AB7500F qPCR efficiencies obtained by testing reference genomic DNA (gDNA) were $90.9 \%, 86.4 \%$, and $94.6 \%$ for $C$. jejuni, $C$. coli, and C. lari, respectively, with all correlation coefficient values $>0.99$. The qPCR results exhibited $100 \%$ specificity by testing gDNA samples from 37 non-target reference strains and 86 target strains (50 C. jejuni, 27 C. coli, and 9 C. lari strains) in this study. The lowest detection level using gDNA was 4, 7, and 2 genome copies per reaction for C. jejuni, C. coli, and $C$. lari, respectively. With a 2-day enrichment procedure, the qPCR method correctly detected target species in a spiked food matrix (frog leg, an aquaculture product). The sensitivity in $25 \mathrm{~g}$ food matrix was 4 colony-forming units (CFUs) for $C$. jejuni, 3 CFUs for $C$. coli, and 2 CFUs for $C$. lari. The results suggest that this AB7500F-based qPCR has potential applications for the identification of C. jejuni, C. coli, and C. lari in contaminated food.
\end{abstract}

Keywords: Campylobacter, species identification, multiplex qPCR, food analysis

\section{Introduction}

A S A LEADING cause of foodborne bacterial gastroenteritis, Campylobacter is responsible for more than 1.3 million infections each year in the United States (CDC, 2020). Campylobacteriosis, the infection of Campylobacter, is often linked to consumption of contaminated meat, poultry, dairy, aquaculture, and fresh produce (Silva et al., 2011; Gharst et al., 2013; Government of Canada, 2020). Besides animal origins, the environmental reservoirs for Campylobacter contamination usually include manure, water, and soil (Whiley et al., 2013). Syndromes of Campylobacteriosis range from abdominal cramps, fever, to bloody diarrhea (CDC, 2020). In high-risk groups, such as young children, pregnant women, and elderly, Campylobacteriosis may cause more severe or even fatal complications (USDA, 2020).
In the genus Campylobacter, Campylobacter jejuni is the most common pathogenic species, whereas Campylobacter coli and Campylobacter lari are recognized with increasing clinical importance (Kaakoush et al., 2015; Saiyudthong et al., 2015). In addition to gastroenteritis, Campylobacter infection is sometimes related to other complications. C. jejuni is a risk factor of Guillan-Barre syndrome, an autoimmune disease (Neal-McKinney et al., 2018); C. coli may cause second trimester intrauterine growth restriction in pregnant women, which leads to abortions (KupermanShani et al., 2015); and C. lari infections may result in life-threatening septicemia (Morishita et al., 2013). Therefore, the capability of rapid, specific, and sensitive identification of C. jejuni, C. coli, and C. lari will contribute to the improvement of food safety to protect public health.

Applied Technology Center at the Pacific Northwest Laboratory (PNL), Office of Regulatory Science, Office of Regulatory Affairs (ORA), the U.S. Food and Drug Administration, Bothell, Washington, USA.

The views presented in this work do not necessarily reflect those of the FDA, nor do we specifically endorse the listed instrumentation or products. 
Previously, a multiplex quantitative polymerase chain reaction (qPCR) utilizing the SmartCycler instrument (Cepheid, Inc., Sunnyvale, CA) was developed in this laboratory to simultaneously detect $C$. jejuni, $C$. coli, and C. lari by using genomic DNA (gDNA) of pure cultures and artificially spiked raw milk without enrichment (Liu et al., 2017). However, the SmartCycler instrument was recently discontinued by its manufacturer.

In this study, a multiplex assay was optimized on the Applied Biosystems 7500 Fast (AB7500F) platform (Thermo Fisher Scientific, Inc., Waltham, MA) to continue using qPCR for simultaneous identification of the target species. Performance of the method on AB7500F was evaluated, including the efficiency, specificity, and sensitivity. In addition to gDNA of pure bacterial cultures, the AB7500F qPCR in combination with an enrichment procedure was also tested with frog leg, a widely consumed aquaculture product regulated by FDA, to assess the applications to the analysis of Campylobacter-contaminated food.

\section{Materials and Methods}

Bacterial strains, culture conditions, and gDNA extraction

All glycerol stocks were kept frozen at $-70^{\circ} \mathrm{C}$ until use. Thirty-seven non-target reference strains in 33 species were obtained from the American Type Culture Collection (ATCC) and cultured under appropriate conditions following the FDA Bacteriological Analytical Manual (BAM) (FDA, 2020a) for DNA extraction. Fourteen target reference strains (four C. jejuni, five C. coli, and five C. lari strains) were obtained from ATCC. Seventy-two Campylobacter field strains (46 C. jejuni, 22 C. coli, and 4 C. lari strains) were from the laboratory collection. They had been previously characterized following BAM (FDA, 2020a) and by VITEK ${ }^{\circledR}$ MS, an automated mass spectrometry system for microbial identification (bioMérieux, Inc., Marcy-l'Étoile, France).

Campylobacter strains were cultured on $\mathrm{BBL}^{\mathrm{TM}}$ Campylobacter Agar (Cat. No. B21727X; Thermo Fisher Scientific, Inc.) at $37-42^{\circ} \mathrm{C}$ for 2 days under microaerophilic condition before gDNA extraction. gDNA was extracted by using the QIAamp ${ }^{\circledR}$ DNA Mini Protocol on a QIAcube ${ }^{\circledR}$ automated instrument (QIAGEN Company, Hilden, Germany) and subsequently quantified with the double-stranded DNA (dsDNA) BR Assay Kit on a Qubit $^{\circledR} 3.0$ fluorimeter (Thermo Fisher Scientific, Inc.).

\section{Oligo sequences and $q P C R$ conditions}

For the detection of $C$. jejuni, C. lari, and the internal amplification control (IAC), sequences were adopted from the SmartCycler qPCR method previously published (Liu et al., 2017). For $C$. coli detection, new oligos were designed by using the PrimerQuest tool (IDT, 2020) to improve qPCR efficiency. Sequences of all primers and probes are listed in Table 1. The IAC template is a $140 \mathrm{bp}$ fragment, including a $114 \mathrm{bp}$ green fluorescence protein (GFP) amplicon and protection bases on both ends: 5'-GTGCTGCTGCCCGAC AACCACTACCTGAGCACCCAGTCCGCCCTGAGCAA AGACCCCAACGAGAAGCGCGATCACATGGTCCTGC TGGAGTTCGTGACCGCCGCCGGGATCACTCTCGGC ATGGACGAGCTGTACAAGTA-3'. The oligos were

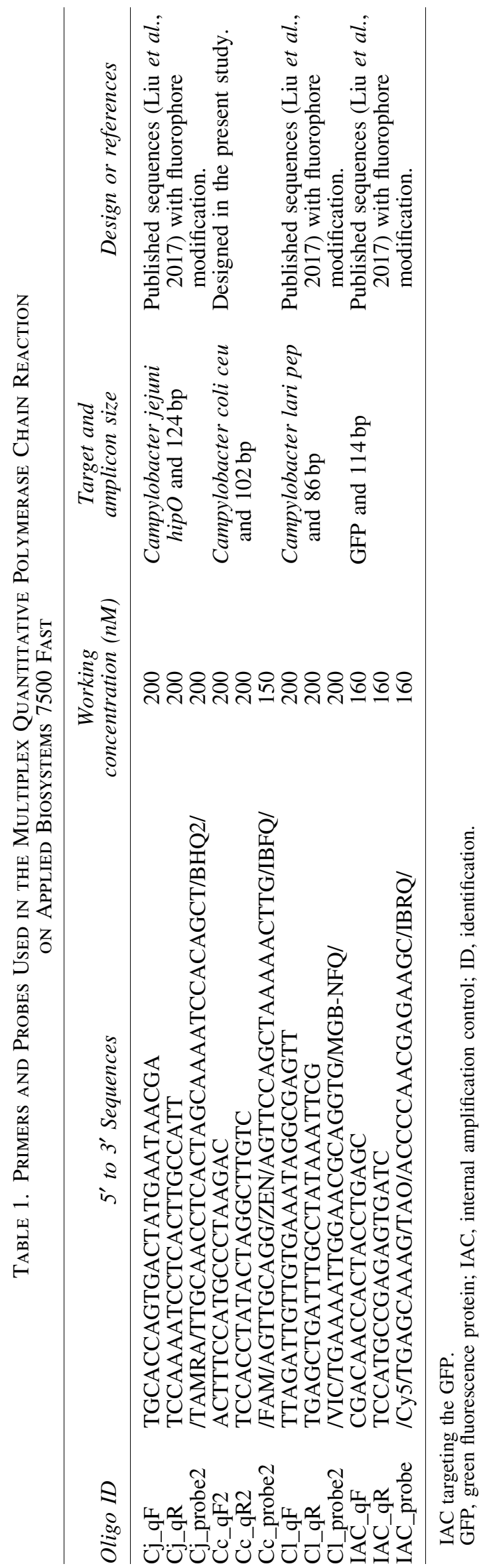


synthesized by Applied Biosystems of Thermo Fisher Scientific, Inc., (Waltham, MA) and Integrated DNA Technologies (Coralville, IA).

AB7500F qPCR reactions were run under fast mode. Each reaction contained $12.5 \mu \mathrm{L}$ TaqMan ${ }^{\mathrm{TM}}$ Universal PCR Master Mix (Cat. No. 4304437; Thermo Fisher Scientific, Inc.), $2.875 \mu \mathrm{L}$ primers and probes targeting the three Campylobacter spp., $1.8 \mu \mathrm{L}$ IAC oligos $(0.4 \mu \mathrm{L}$ primers, $0.4 \mu \mathrm{L}$ probe, and $1.0 \mu \mathrm{L}$ IAC template), $0.2 \mu \mathrm{L}$ Platinum ${ }^{\mathrm{TM}}$ Taq Hot Start DNA Polymerase (Cat. No. 10966018; Thermo Fisher Scientific, Inc.), and molecular biology grade nuclease-free water to reach a final volume of $25 \mu \mathrm{L}$. Working concentrations of primers and probes are listed in Table 1. The final concentration of IAC template was $0.6 \mathrm{pM}$, containing $\sim 3.6 \times 10^{5}$ copies of the GFP fragment.

The thermocycling conditions included an initial heat activation step at $95^{\circ} \mathrm{C}$ for $20 \mathrm{~s}$, followed by 45 cycles of denaturation at $95^{\circ} \mathrm{C}$ for $3 \mathrm{~s}$, and annealing and extension at $62^{\circ} \mathrm{C}$ for $30 \mathrm{~s}$ with optics on. The threshold and baseline were automatically calculated by the AB7500F software version 2.3 .

\section{Determination of $q P C R$ efficiency and correlation coefficient}

To determine qPCR efficiency and correlation coefficient, 10-fold serial dilutions of gDNA of ATCC strains were prepared across five orders of magnitude. The amplification efficiency of each target was calculated with an online calculator (Agilent, 2020). The correlation coefficient, $R^{2}$, was determined with Microsoft Excel 2010 by using triplicated qPCR data at each template concentration from a single qPCR run. Copy numbers were calculated with an online tool (URI, 2020). The genome size of Campylobacter was $\sim 1.7 \mathrm{Mb}$ according to the National Center for Biotechnology Information (NCBI, 2020) and published results (Miller et al., 2014; Zhao et al., 2016).

\section{Exclusivity and inclusivity studies}

The exclusivity study was performed by using gDNA of 30 non-Campylobacter reference strains from 29 species in 23 genera, as well as 7 ATCC strains from 4 non-target species in the genus Campylobacter (Campylobacter fetus, Campylobacter iguaniorum, Campylobacter hyointestinalis, and Campylobacter upsaliensis). The inclusivity study was performed by using gDNA of $50 C$. jejuni, $27 C$. coli, and 9 $C$. lari strains, including ATCC references, characterized field isolates from food and environment, and archived clinical isolates transferred from hospitals. Triplicate qPCR experiments were carried out with $\sim 2$ pg gDNA per reaction, which contained $\sim 1000$ Campylobacter genome copies following a standard protocol (FDA, 2020b). The IAC cycle thresholds were usually no more than 30 by using 2 pg gDNA templates. If both targets and IAC were negative, the results were not valid and experiments were repeated. Mean and standard deviation were calculated from qPCR triplicates.

\section{Determination of fractional detection levels}

Tenfold serial dilutions of gDNA were prepared for high concentrations of template $(\sim 300$ genome copies per reaction and above), and twofold serial dilutions were prepared for template concentrations lower than 300 genome copies per reaction. Five qPCR replicates were carried out at each concentration. If the $\mathrm{qPCR}$ results of five replicates were partially positive (i.e., 1-4 positives), the concentration of the template was determined to be the fractional detection level (FDA, 2020c).

\section{The spike-and-recovery study}

Bone-in frog legs were purchased at a local grocery store. Aliquots of $25 \mathrm{~g}$ of frog legs (bone-in) were sterilely placed in Combibag $^{\mathrm{TM}}$ (No. 30551; BioMerieux SKU) and kept frozen until use. The spiking, aging, and recovery experiments were designed following FDA guidelines (FDA, 2020c) and BAM (FDA, 2020a) with minor modifications.

Briefly, fresh Campylobacter cells grown on BBL Campylobacter agar plates were suspended in sterile $1 \times$ phosphatebuffered saline. Quantification of cell suspensions was carried out by optical density measurement on a NanoDrop ${ }^{\text {TM }} 2000$ spectrophotometer. Suspensions were diluted between $10^{-1}$ and $10^{-8}$ times in triplicates. Ten-time E. coli ATCC 35150 were added to one aliquot of Campylobacter suspension at each concentration as the background flora. Frog legs spiked with suspensions containing Campylobacter and background flora were subsequently aged at $4^{\circ} \mathrm{C}$ for 2 days following a standard protocol (FDA, 2020c). Meanwhile, the remaining two aliquots of Campylobacter suspension at each concentration (without background flora) were independently spread on two BBL Campylobacter agar plates for colony counting.

After aging, $225 \mathrm{~mL}$ blood-free Bolton Broth was added to each Combibag containing spiked frog legs. The samples were incubated under microaerophilic condition at $37^{\circ} \mathrm{C}$ without shaking for $2 \mathrm{~h}$, and they were then transferred to $42^{\circ} \mathrm{C}$ for 2 days with a shaking speed of 80 revolutions per minute. After enrichment, $1.0 \mu \mathrm{L}$ filtered culture was directly tested by the AB7500F qPCR method. Another $1.0 \mu \mathrm{L}$ filtered enrichment culture was streaked on selective Bolton Broth with 5\% sheep blood agar and grown under microaerophilic condition as mentioned earlier. Two isolated colonies from each enrichment, which were morphologically like Campylobacter, were examined by using the VITEK MS microbial identification (ID) system with IVD database v3.2.0 (bioMérieux, Inc.) following the manufacturer's instructions to verify the species of recovered bacteria. For each target species, the spike-and-recovery experiment was independently carried out by using two ATCC strains.

\section{Results}

In this study, a multiplex qPCR method was optimized on the AB7500F platform to continue the simultaneous identification for $C$. jejuni, $C$. coli, and $C$. lari after the discontinuation of SmartCycler. The amplification efficiencies of qPCR were $90.9 \%, 86.4 \%$, and $94.6 \%$ for C. jejuni, C. coli, and $C$. lari, respectively. The correlation coefficients of linearity were all $>0.99$ with template concentrations across five orders of magnitude (Fig. 1).

Detection limit of the qPCR method was first evaluated by using gDNA of two ATCC strains from each target species, and similar results were obtained within the same species (Table 2). The lowest fractional level of detection was 4 genome copies for $C$. jejuni, 7 genome copies for $C$. coli, and 2 genome copies for $C$. lari gDNA per reaction. 
A

\section{C. jejuni}

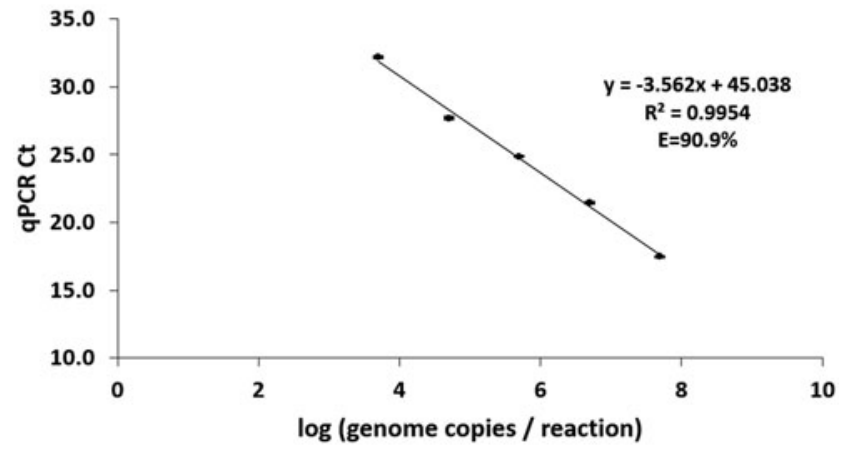

B

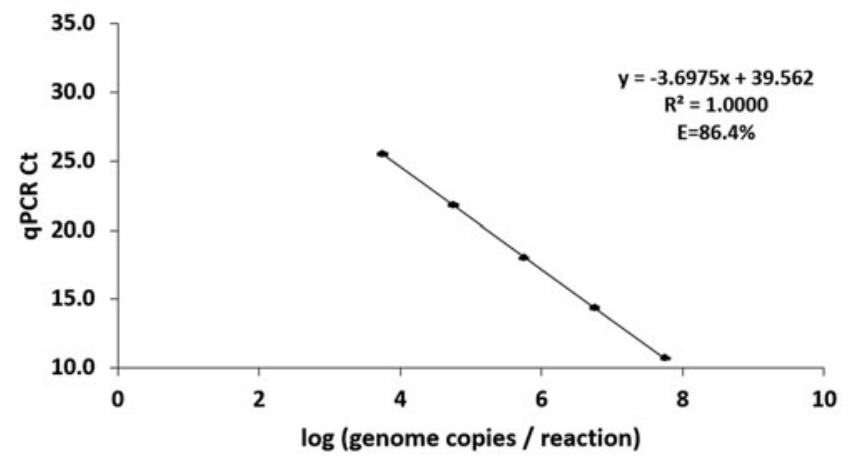

C

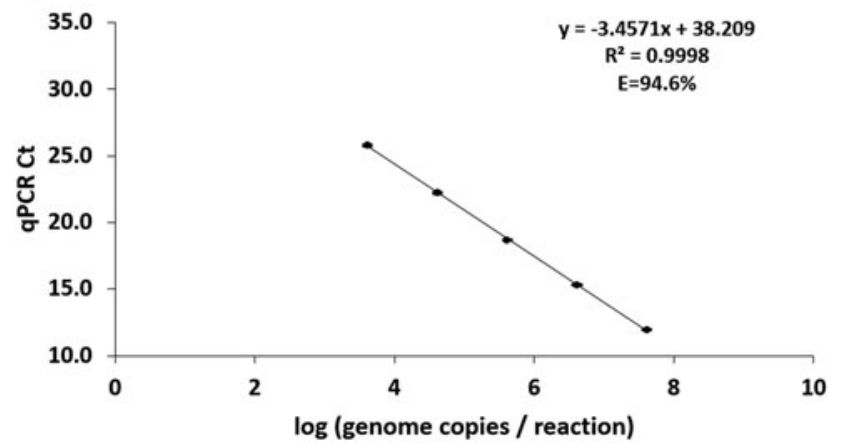

FIG. 1. The qPCR efficiency and correlation coefficient of standard curves for (A) Campylobacter jejuni, (B) Campylobacter coli, and (C) Campylobacter lari determined by using gDNA of ATCC strains. $X$ axis, $\log _{10}$ of genome copies per qPCR reaction. $Y$ axis, qPCR Ct. Each error bar represents the standard deviation of five experimental replicates. Inset, linear regression, correlation coefficient $\left(R^{2}\right)$, and efficiency (E). ATCC, American Type Culture Collection; $\mathrm{Ct}$, cycle threshold; gDNA, genomic DNA; qPCR, quantitative polymerase chain reaction.

Using gDNA of 37 non-target ATCC strains (Table 3) as templates, this qPCR method showed $100 \%$ exclusive specificity. There was no false positive detected, clearly showing that the method did not cross-react with the non-target species tested in the study. As all IAC results were acceptable, false negatives caused by PCR inhibition could be excluded. Notably, qPCR results were all negative when testing gDNA of seven ATCC strains from non-target Campylobacter spp., C. fetus, C. hyointestinalis, C. iguaniorum, and
Table 2. Detection Limit Determined by Using Genomic DNA of American Type Culture COLlection Strains

\begin{tabular}{lcc}
\hline Target species & $\begin{array}{c}\text { ATCCNo. } \\
\text { of strains }\end{array}$ & $\begin{array}{c}\text { Lowest detection level } \\
\text { (genome copies per reaction) }\end{array}$ \\
\hline Campylobacter & 33291 & 4 \\
jejuni & 33560 & 6 \\
Campylobacter & 33559 & 7 \\
coli & 43133 & 10 \\
Campylobacter & 35222 & 2 \\
lari & 43675 & 3 \\
\hline \multicolumn{2}{l}{ Genome copies were rounded up to the next nearest integers. } \\
ATCC, American Type Culture Collection.
\end{tabular}

C. upsaliensis. The results demonstrate that this method is species specific within the genus Campylobacter.

Both ATCC strains and previously characterized field strains were used in the inclusivity study. Results showed that this qPCR method achieved 0 false negative by testing gDNA of 50 C. jejuni, 27 C. coli, and 9 C. lari strains (Table 4).

Table 3. Reference Strains Used IN THE EXCLUSIVITY STUDY

\begin{tabular}{|c|c|c|}
\hline Genus & Species & ATCC No. \\
\hline Acinetobacter & baumanni & 19606 \\
\hline Aeromonas & hydrophila & 7965 \\
\hline Alcaligenes & faecalis & 8750 \\
\hline Bacillus & cereus & 14579 \\
\hline Bacillus & subtilis & 19659 \\
\hline Bordetella & bronchiseptica & 10580 \\
\hline Burkholderia & cepacia & 25608 \\
\hline Enterobacter & aerogenes & 13048 \\
\hline Enterococcus & faecalis & 29212 \\
\hline Enterococcus & durans & 6056 \\
\hline Escherichia & coli & 25922 \\
\hline Escherichia & coli & 35150 \\
\hline Hafnia & alvei & 13337 \\
\hline Klebsiella & pneumoniae & 13883 \\
\hline Leclercia & adecarboxylata & 23216 \\
\hline Listeria & monocytogenes & 19111 \\
\hline Listeria & seeligeri & 35967 \\
\hline Pasteurella & aerogenes & 27883 \\
\hline Plesiomonas & shigelloides & 51903 \\
\hline Proteus & mirabilis & 7002 \\
\hline Providencia & rettgeri & 35565 \\
\hline Providencia & alcalifaciens & 51902 \\
\hline Salmonella & cholerasuis & 6539 \\
\hline Salmonella & bongori & 43975 \\
\hline Serratia & liquifaciens & 27592 \\
\hline Staphylococcus & xylosus & 29971 \\
\hline Streptococcus & bovis & 9809 \\
\hline Streptococcus & pyogenes & 19615 \\
\hline Vibrio & cholerae & 15748 \\
\hline Yarrowia & lipolytica & 9773 \\
\hline Campylobacter & fetus & 25936 \\
\hline Campylobacter & fetus & 19438 \\
\hline Campylobacter & fetus & 33561 \\
\hline Campylobacter & fetus & 27374 \\
\hline Campylobacter & iguaniorum & BAA2680 \\
\hline Campylobacter & hyointestinalis & 35217 \\
\hline Campylobacter & upsaliensis & 49816 \\
\hline
\end{tabular}

ATCC, American Type Culture Collection. 
Table 4. American Type Culture Collection

ReFERENCE Strains and Field IsOlates Used

IN THE INCLUSIVITY STUDY FOR CAMPYLOBACTER

JEJUNI, CAMPYLOBACTER COLI, AND CAMPYLOBACTER LARI IDENTIFICATION

Campylobacter spp. ATCC or PNL No.

C. jejuni

C. jejuni

C. jejuni

C. jejuni

C. jejuni

C. jejuni ${ }^{\mathrm{a}}$

C. jejuni $i^{\mathrm{a}}$

C. jejuni $i^{\mathrm{a}}$

C. jejuni ${ }^{\mathrm{a}}$

C. jejuni ${ }^{\mathrm{a}}$

C. jejuni ${ }^{\mathrm{a}}$

C. jejuni ${ }^{\mathrm{a}}$

C. jejuni ${ }^{\mathrm{a}}$

C. jejuni ${ }^{\mathrm{a}}$

C. jejuni ${ }^{\mathrm{a}}$

C. jejuni ${ }^{\mathrm{a}}$

C. jejuni ${ }^{\mathrm{a}}$

C. jejuni ${ }^{\mathrm{a}}$

C. jejuni ${ }^{\mathrm{a}}$

C. jejuni ${ }^{\mathrm{a}}$

C. jejuni ${ }^{\mathrm{a}}$

C. jejuni ${ }^{\mathrm{a}}$

C. jejuni ${ }^{\mathrm{a}}$

C. jejuni ${ }^{\mathrm{a}}$

C. jejuni ${ }^{\mathrm{a}}$

C. jejuni ${ }^{\mathrm{a}}$

C. jejuni ${ }^{\mathrm{a}}$

C. jejuni ${ }^{\mathrm{a}}$

C. jejuni

C. jejuni

C. jejuni

C. jejuni

C. jejuni

C. jejuni

C. jejuni

C. jejuni

C. jejuni

C. jejuni

C. jejuni

C. jejuni

C. jejuni

C. jejuni

C. jejuni

C. jejuni

C. jejuni

C. jejuni

C. jejuni

C. jejuni

C. jejuni

C. jejuni

C. jejuni

C. coli

C. coli

C. coli

C. coli

C. coli

C. coli
TABLE 4. (CONTINUED)

\begin{tabular}{|c|c|}
\hline Campylobacter spp. & ATCC or PNL No. \\
\hline C. coli $^{\mathrm{b}}$ & 20G12 \\
\hline C. coli & $21 \mathrm{~A}-34$ \\
\hline C. coli & $21 \mathrm{~F}-42$ \\
\hline C. coli & $20 \mathrm{~A} 64$ \\
\hline C. coli & $20 \mathrm{~A} 55$ \\
\hline C. coli & $21 \mathrm{~B} 14$ \\
\hline C. coli & 21B52 \\
\hline C. coli & 21B53 \\
\hline C. coli & $21 \mathrm{~B} 62$ \\
\hline C. coli & $21 \mathrm{~F} 34$ \\
\hline C. coli & R11B1-06 \\
\hline C. coli & R11B1-13 \\
\hline C. coli & R11B1-19 \\
\hline C. coli & R11B1-21 \\
\hline C. coli & R11B5-33 \\
\hline C. coli & R4B2-10 \\
\hline C. coli & R4B2-15 \\
\hline C. coli & R4B2-19 \\
\hline C. coli & R4B2-31 \\
\hline C. coli & R7B1-44 \\
\hline C. coli & R7B3-75 \\
\hline C. $\operatorname{coli}^{\mathrm{c}}$ & N41652 \\
\hline \multicolumn{2}{|l|}{ C. lari } \\
\hline C. lari & ATCC 35221 \\
\hline C. lari & ATCC 35222 \\
\hline C. lari & ATCC 43675 \\
\hline C. lari & ATCC BAA 1060 \\
\hline C. lari & ATCC 35223 \\
\hline C. lari & R7B2-71 \\
\hline C. lari & R7B5-45 \\
\hline C. lari & $20 \mathrm{~A} 31$ \\
\hline C. lari & R11B5-11 \\
\hline
\end{tabular}

${ }^{\mathrm{a}}$ Speciation of the field strains was previously published (Gharst et al., 2013).

${ }^{\mathrm{b}}$ Speciation of the field strain was previously published (Liu et al., 2016).

${ }^{\mathrm{c}}$ Speciation of the field strain was previously published (Zhao et al., 2016).

ATCC, American Type Culture Collection; PNL, Pacific Northwest Laboratory.

The AB7500F qPCR method was also evaluated by using artificially mixed gDNA of the target species $(C$. jejuni ATCC 33291, C. coli ATCC 43479, and C. lari ATCC 35222 mixed at $1 \mathrm{pg} / \mathrm{species} /$ reaction). Positive qPCR results with acceptable IAC demonstrated that this method simultaneously identified gDNA of all three target species in a single reaction (Table 5). A set of representative multiplex amplification plots is shown in Figure 2.

After assessing the multiplex qPCR with gDNA of pure cultures, artificially contaminated frog legs were used to mimic real food contaminations. Results of the spike-andrecovery study showed that this qPCR method was able to correctly identify spiked C. jejuni, C. coli, and C. lari in frog legs. The lowest detection level was 4, 3, and 2 spiked colony-forming units (CFUs) per $25 \mathrm{~g}$ food for $C$. jejuni, $C$. coli, and C. lari, respectively. Culture of frog legs spiked with background flora but without Campylobacter spiking (blank control) yielded negative qPCR results with acceptable IAC, clearly showing that the positive qPCR signals detected in spiked food matrix came from target Campylobacter spp. 
Table 5. Quantitative Polymerase Chain Reaction Results by Using Artificially Mixed Genomic DNA of the Three Target Species

\begin{tabular}{|c|c|c|c|c|c|c|c|c|}
\hline Sample & $\begin{array}{l}\text { Mean } \\
\text { C. j Ct }\end{array}$ & $\begin{array}{l}S D \\
\text { C. } \mathrm{j} C t\end{array}$ & $\begin{array}{l}\text { Mean } \\
\text { C. c } C t\end{array}$ & $\begin{array}{l}S D \\
\text { C. c } C t\end{array}$ & $\begin{array}{l}\text { Mean } \\
\text { C. } 1 C t\end{array}$ & $\begin{array}{l}S D \\
\text { C. } 1 C t\end{array}$ & $\begin{array}{l}\text { Mean } \\
\text { IAC Ct }\end{array}$ & $\begin{array}{l}S D \\
I A C C t\end{array}$ \\
\hline Mixed gDNA & 28.80 & 0.12 & 25.81 & 0.06 & 28.05 & 0.18 & 27.36 & 0.37 \\
\hline $\begin{array}{l}\text { qPCR negative control } \\
\left(\mathrm{ddH}_{2} \mathrm{O}\right)\end{array}$ & NA & NA & NA & NA & NA & NA & 27.42 & 0.15 \\
\hline
\end{tabular}

Mean and SD were calculated from triplicates.

C. c, Campylobacter coli; C. j, Campylobacter jejuni; C. l, Campylobacter lari; Ct, cycle threshold; gDNA, genomic DNA; IAC, internal amplification control; NA, not applicable; qPCR, quantitative polymerase chain reaction; SD, standard deviation.

instead of the matrix itself. In addition, the results by using the VITEK MS microbial ID system confirmed that the Campylobacter-like isolates post-enrichment came from the spiked species whereas there was no colony isolated from the blank control (Table 6).

\section{Discussion}

qPCR has great advantages over traditional PCR (Valasek and Repa, 2005). We have previously developed a SmartCycler-based qPCR method, which allows simultaneous identification for $C$. jejuni, $C$. coli, and $C$. lari in a single reaction (Liu et al., 2017). To address the recent discontinuation of the SmartCycler by its manufacturer, a follow-up study is reported here with an optimized multiplex qPCR on $\mathrm{AB} 7500 \mathrm{~F}$ as the replacement. Widely used in laboratories, the AB7500F system has greater capacity than the SmartCycler (96 wells vs. 16 wells per instrument). Here, a unique com- bination of target genes was employed for Campylobacter spp. identification ( $h i p O$ for $C$. jejuni, ceu for C. coli, and pep for $C$. lair). Performance of the new method was similar to the SmartCycler method regarding qPCR specificity (100\% exclusivity and inclusivity on both platforms with the stains tested) and sensitivity (4, 7, and 2 genome copies for $C$. jejuni, C. coli, and C. lari, respectively, on the AB7500F platform vs. 2, 3, and 2 genome copies on the SmartCycler platform).

In this study, the strains used for exclusivity test were the same as those used for the SmartCycler method (30 nonCampylobacter ATCC strains from 29 species in 23 genera, as well as 7 ATCC strains from 4 non-target Campylobacter spp. C. fetus, C. iguaniorum, C. hyointestinalis, and C. upsaliensis). Notably, compared with the 32 Campylobacter strains used for evaluation of the SmartCycler qPCR, more field strains were used for the inclusivity test in this study, resulting in a total of 86 target strains (50 from C. jejuni, 27 from $C$. coli, and 9 from $C$. lari). No cross-reactivity was
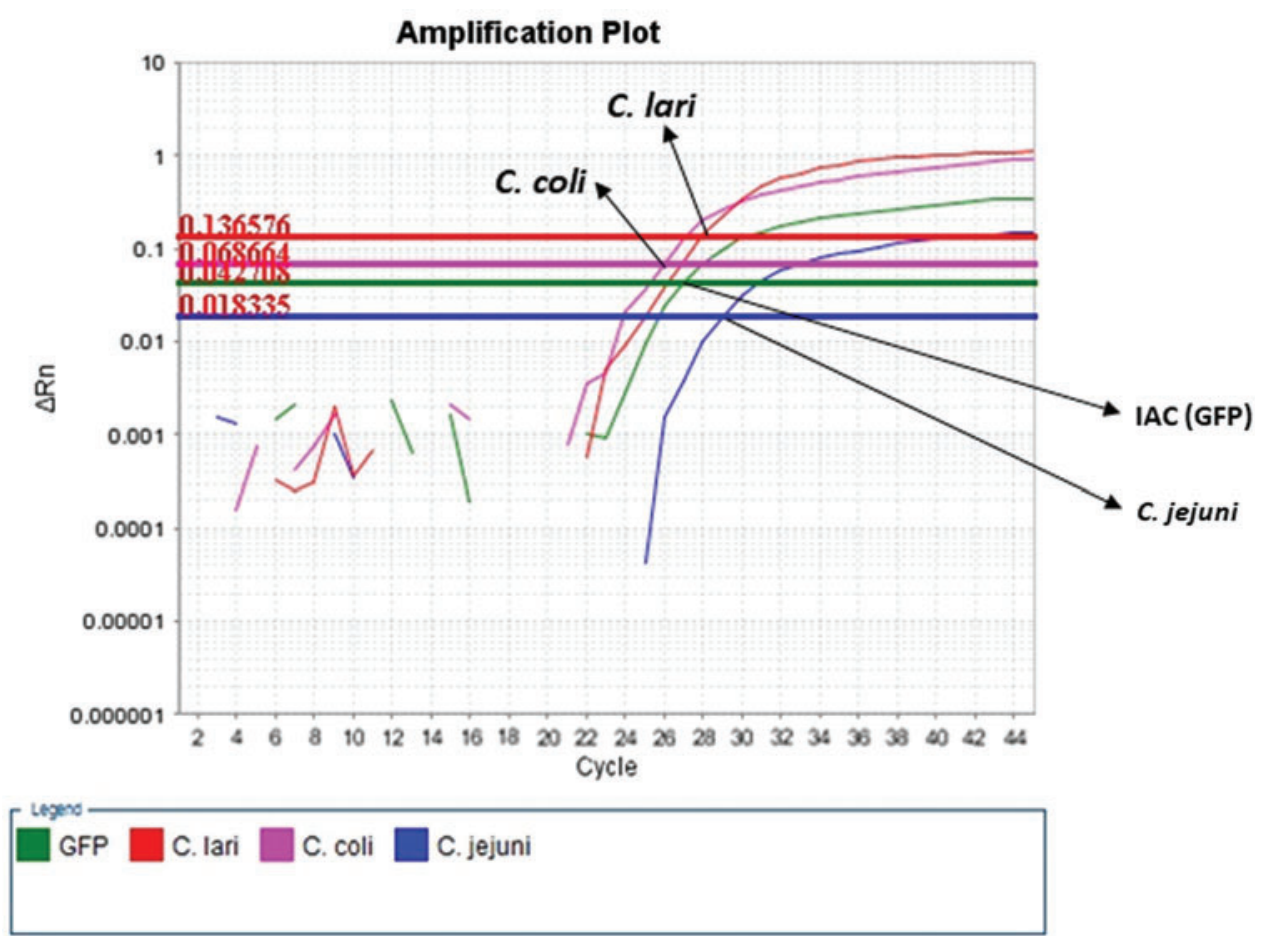

FIG. 2. Representative multiplex qPCR amplification plots using 1:1:1 artificially mixed gDNA of Campylobacter jejuni, Campylobacter coli, and Campylobacter lari strains. $X$ axis, qPCR cycle number. $Y$ axis, delta Rn, normalized fluorescence value subtracted by baseline. Arrows indicate the $\mathrm{Ct}$ values of $C$. jejuni, $C$. coli, $C$. lari, and IAC in this experiment. Ct, cycle threshold; gDNA, genomic DNA; IAC, internal amplification control; qPCR, quantitative polymerase chain reaction. Color images are available online. 
Table 6. Results of a Representative SpIKe-And-Recovery Experiment

\begin{tabular}{|c|c|c|c|c|c|c|}
\hline Sample description & Spiked strains & $\begin{array}{c}\text { Lowest } \\
\text { detection level } \\
(C F U s / 25 \mathrm{~g})\end{array}$ & Target $C t$ & $I A C C t$ & $\begin{array}{l}\text { Correct ID } \\
\text { by } q P C R \text { ? }\end{array}$ & $\begin{array}{c}\text { Confirmation } \\
\text { by VITEK }{ }^{\mathbb{B}} M S\end{array}$ \\
\hline $25 \mathrm{~g}$ Frog leg spike & $\begin{array}{r}\text { Campylobacter } \\
\text { jejuni } 33291\end{array}$ & 4 & C. $j=28.24$ & 30.05 & Yes & C. jejuni \\
\hline 25 g Frog leg spike & $\begin{array}{l}\text { Campylobacter } \\
\text { coli } 43485\end{array}$ & 3 & C. $c=21.45$ & 30.09 & Yes & C. coli \\
\hline 25 g Frog leg spike & $\begin{array}{c}\text { Campylobacter } \\
\text { lari } 35223\end{array}$ & 2 & C. $l=22.93$ & 32.21 & Yes & C. lari \\
\hline $\begin{array}{l}25 \text { g Frog leg } \\
\text { (spike blank } \\
\text { control) }\end{array}$ & NA & NA & Undetermined & 31.17 & NA & NA \\
\hline $\begin{array}{l}\text { qPCR positive } \\
\text { control (ATCC } \\
\text { gDNA) }\end{array}$ & NA & NA & $\begin{array}{l}\text { C. } j=24.02 ; \\
\quad \text { C. } c=23.01 ; \\
\quad \text { C. } l=24.18\end{array}$ & 34.99 & Yes & NA \\
\hline $\begin{array}{l}\text { qPCR negative } \\
\text { control }\left(\mathrm{ddH}_{2} \mathrm{O}\right)\end{array}$ & NA & NA & Undetermined & 29.12 & NA & NA \\
\hline
\end{tabular}

Lowest detection level was rounded to the next integer.

ATCC, American Type Culture Collection; $C . c, C$. coli; $C . j, C$. jejuni; $C$. l, C. lari; CFU, colony-forming unit; Ct, cycle threshold; gDNA, genomic DNA; IAC, internal amplification control; ID, identification; NA, not applicable; qPCR, quantitative polymerase chain reaction.

detected at the species level by using gDNA of a wide range of other bacterial genera or non-target species in the same genus Campylobacter. All the 86 target strains, including 14 ATCC references and 72 field strains, were all correctly identified by the AB7500F-based multiplex qPCR method, indicating potential applications of this method to surveillance studies and outbreak investigations associated with Campylobacter food poisoning.

Typically, qPCR efficiency lies between $90 \%$ and $110 \%$. In this study, after optimization on target gene, primer concentration, annealing temperature, and concentration of Taq polymerase, the efficiency of $C$. coli amplification was $86.4 \%$ (Fig. 1B, inset). However, satisfactory results were obtained for specific and sensitive identification of the target species by using gDNA from pure cultures or spiked food. Better qPCR performance might be achieved in future studies with higher efficiency after more systematic optimizations while keeping detection of the other two species uncompromised.

In addition to the assessment with gDNA of pure cultures, artificially contaminated frog legs were used for method evaluation to mimic real conditions. Edible frog is an aquatic animal product regulated by FDA (2017) and is widely consumed by people in Europe, Asia, Africa, and the United States (Onadeko et al., 2011; Akinyemi and Ogaga, 2015; NOAA, 2017). In 2017, 3080 metric tons of edible frogs were imported to the United States for food consumption with a value of \$12.5 million (NOAA, 2017).

Campylobacter-like bacteria have been isolated from northern leopard frogs (Gossling et al., 1982). A C. fetus infection is associated with consumption of frog meat $(\mathrm{Pa}-$ trick et al., 2013). Campylobacter spp. are also found in surface water (Nilsson et al., 2017, 2018; WHO, 2019) and other aquacultures (Soonthornchaikul and Garelick, 2009). Therefore, there is possibility that edible frogs are contaminated by $C$. jejuni, $C$. coli, and $C$. lari, which may result in sporadic infections and outbreaks threatening public health. The AB7500F-based qPCR assay and the enrichment procedure developed here have laid the foundation to identify $C$. jejuni, C. coli, and C. lari from contaminated foods and ensure the United States' food safety.

As background flora in food matrices have been shown to inhibit growth of target bacteria (Vold et al., 2000; Lardeux et al., 2015; Biesta-Peters et al., 2019), enrichment procedures specific for Campylobacter recovery are important for food examinations. In this study, an effective procedure was developed to recover $C$. jejuni, $C$. coli, and $C$. lari at low abundance from refrigerated bone-in frog legs. Even after aging at $4^{\circ} \mathrm{C}$ for 2 days, the AB7500F qPCR method was able to correctly detect the target species from 10-time background flora. The lowest detection levels were 2-4 CFUs per $25 \mathrm{~g}$ food matrix, which were similar to those obtained by using gDNA of pure cultures (Table 6 vs. Table 2). In addition, the VITEK MS results confirmed that the Campylobacter-like colonies isolated from enrichment cultures were the spiked species (Table 6), showing that this enrichment procedure facilitates the recovery of target Campylobacter spp. more than bacterial flora in spiked frog legs. The unique combination of $\mathrm{GasPak}^{\mathrm{TM}}$ sachet for microaerophilic growth, Combibag for food handling, and BBL Campylobacter Agar for colony isolation ensured the traceability and improved the ease of use in food safety laboratories.

In summary, this qPCR method in combination with the enrichment procedure shows great potential as a rapid, specific, and sensitive diagnostic tool for both pure cultures and foods contaminated with $C$. jejuni, $C$. coli, and $C$. lari.

\section{Acknowledgments}

The author thanks Dr. Jason Neal-McKinney for technical assistance and discussion; Drs. Wen-Hsin Cindy Wu, Jinxin $\mathrm{Hu}$, Dan Rice, and Karen Jinneman for scientific discussion. Technical support from the Microbiology Branch of Pacific Northwest Laboratory (PNL) is greatly appreciated. The author would like to thank Dr. Shaohua Zhao at the FDA Center for Veterinary Medicine for providing a published $C$. coli strain. Finally, the author is grateful to the PNL 
management team and FDA/ORA/ORS/Office of Research Coordination, Evaluation and Training (ORCET) for review and support.

\section{Disclosure Statement}

No competing financial interests exist.

\section{Funding Information}

No funding was received for this article.

\section{References}

Agilent. qPCR Standard Curve Slope to Efficiency Calculator. 2020. Available at: www.genomics.agilent.com/biocalcula tors/calcSlopeEfficiency.jsp, accessed September 9, 2020.

Akinyemi AF, Ogaga ED. Frog consumption pattern in Ibadan, Nigeria. J Stud Manage Plan 2015;1:522-531.

Biesta-Peters EG, Jongenburger I, De Boer E, Jacobs-Reitsma WF. Validation by interlaboratory trials of EN ISO 10272 Microbiology of the food chain-Horizontal method for detection and enumeration of Campylobacter spp.-Part 1: Detection method. Int J Food Microbiol 2019;288:39-46.

[CDC] Centers for Disease Control and Prevention. Centers for Disease Control and Prevention: Campylobacter. 2020. Available at: www.cdc.gov, accessed September 9, 2020.

[FDA] U.S. Food and Drug Administration. FDA Seafood List Updates. 2017. Available at: https://www.fda.gov/food/sea food-guidance-documents-regulatory-information/fda-seafoodlist-updates-2017, accessed September 9, 2020.

[FDA] U.S. Food and Drug Administration. Bacteriological Analytical Manual. 2020a. Available at: www.fda.gov/Food/ FoodScienceResearch/LaboratoryMethods/ucm2006949.htm, accessed September 9, 2020.

[FDA] U.S. Food and Drug Administration. FDA OFVM MiSeq WGS protocols. 2020b.

[FDA] U.S. Food and Drug Administration. Guidelines for the Validation of Analytical Methods for the Detection of Microbial Pathogens in Foods and Feeds $3^{\text {rd }}$ Edition. 2020c. Available at: https://www.fda.gov/media/83812/download, accessed September 9, 2020.

Gharst G, Oyarzabal OA, Hussain SK. Review of current methodologies to isolate and identify Campylobacter spp. from foods. J Microbiol Methods 2013;95:84-92.

Gossling J, Loesche WJ, Nace GW. Large intestine bacterial flora of nonhibernating and hibernating leopard frogs (Rana pipiens). Appl Environ Microbiol 1982;44:59-66.

Government of Canada. The Public Health Agency of Canada: Campylobacter jejuni. 2020. Available at: https://www .canada.ca/en/public-health/services/food-poisoning/campylo bacter-jejuni.html, accessed September 9, 2020.

[IDT] Integrated DNA Technologies. The PrimerQuest Tool. 2020. Available at: www.idtdna.com/Primerquest/Home/ Index, accessed September 9, 2020.

Kaakoush NO, Castano-Rodriguez N, Mitchell HM, Man SM. Global epidemiology of Campylobacter infection. Clin Microbiol Rev 2015;28:687-720.

Kuperman-Shani A, Vaknin Z, Mendlovic S, Zaidenstein R, Melcer Y, Maymon R. Campylobacter coli infection causing second trimester intrauterine growth restriction (IUGR): A case report and review of the literature. Prenat Diagn 2015; 35:1258-1261.
Lardeux AL, Guillier L, Brasseur E, Doux C, Gautier J, Gnanou-Besse N. Impact of the contamination level and the background flora on the growth of Listeria monocytogenes in ready-to-eat diced poultry. Lett Appl Microbiol 2015;60: 481-490.

Liu KC, Jinneman KC, Neal-Mckinney J, Wu WH, Rice DH. Genome sequencing and annotation of a Campylobacter coli strain isolated from milk with multidrug resistance. Genom Data 2016;8:123-125.

Liu KC, Jinneman KC, Neal-Mckinney J, Wu WH, Rice DH. Simultaneous identification of Campylobacter jejuni, Campylobacter coli, and Campylobacter lari with SmartCyclerbased multiplex quantitative polymerase chain reaction. Foodborne Pathog Dis 2017;14:371-378.

Miller WG, Yee E, Chapman MH, Smith TP, Bono JL, Huynh S, Parker CT, Vandamme P, Luong K, Korlach J. Comparative genomics of the Campylobacter lari group. Genome Biol Evol 2014;6:3252-3266.

Morishita S, Fujiwara H, Murota H, Maeda Y, Hara A, Horii T. Bloodstream infection caused by Campylobacter lari. J Infect Chemother 2013;19:333-337.

[NCBI] National Center for Biotechnology Information. Campylobacter Genome. 2020. Available at: https://www .ncbi.nlm.nih.gov, accessed September 9, 2020.

Neal-Mckinney JM, Liu KC, Jinneman KC, Wu WH, Rice DH. Whole genome sequencing and multiplex qPCR methods to identify Campylobacter jejuni encoding cst-II or cst-III sialyltransferase. Front Microbiol 2018;9:408.

Nilsson A, Johansson C, Skarp A, Kaden R, Engstrand L, Rautelin H. Genomic and phenotypic characteristics of Swedish C. jejuni water isolates. PLoS One 2017;12:e0189222.

Nilsson A, Skarp A, Johansson C, Kaden R, Engstrand L, Rautelin H. Characterization of Swedish Campylobacter coli, clade 2 and clade 3 water isolates. Microbiologyopen 2018;7: e00583.

[NOAA] National Oceanic and Atmospheric Administration. National Oceanic and Atmospheric Administration Fisheries: Imports and Exports of Fishery Products Annual Summary, 2017. 2017. Available at: https://www.st.nmfs.noaa.gov/Assets/ commercial/trade/Trade2017.pdf, accessed September 9, 2020.

Onadeko AB, Egonmwan RI, Saliu JK. Edible amphibian species: Local knowledge of their consumption in Southwest Nigeria and their nutritional value. West Afr J Appl Ecol 2011;19:67-76.

Patrick ME, Gilbert MJ, Blaser MJ, Tauxe RV, Wagenaar JA, Fitzgerald C. Human infections with new subspecies of Campylobacter fetus. Emerg Infect Dis 2013;19:1678-1680. Saiyudthong S, Phusri K, Buates S. Rapid detection of Campylobacter jejuni, Campylobacter coli, and Campylobacter lari in fresh chicken meat and by-products in Bangkok, Thailand, using modified multiplex PCR. J Food Prot 2015; 78:1363-1369.

Silva J, Leite D, Fernandes M, Mena C, Gibbs PA, Teixeira P. Campylobacter spp. as a foodborne pathogen: A review. Front Microbiol 2011;2:200.

Soonthornchaikul N, Garelick H. Antimicrobial resistance of Campylobacter species isolated from edible bivalve molluscs purchased from Bangkok markets, Thailand. Foodborne Pathog Dis 2009;6:947-951.

[URI] The University of Rhode Island. Copynumber Calculator of URI Genomics \& Sequencing Center. 2020. Available at: http://cels.uri.edu/gsc/cndna.html, accessed September 9, 2020. 
[USDA] U.S. Department of Agriculture. USDA Food Safety and Inspection Service: Campylobacter. 2020. Available at: https:// www.fsis.usda.gov/wps/wcm/connect/fsis-content/internet/main/ topics/food-safety-education/get-answers/food-safety-factsheets/foodborne-illness-and-disease/campylobacter-questionsand-answers/ct_index, accessed September 9, 2020.

Valasek MA, Repa JJ. The power of real-time PCR. Adv Physiol Educ 2005;29:151-159.

Vold L, Holck A, Wasteson Y, Nissen H. High levels of background flora inhibits growth of Escherichia coli O157:H7 in ground beef. Int J Food Microbiol 2000;56: 219-225.

Whiley H, Van Den Akker B, Giglio S, Bentham R. The role of environmental reservoirs in human campylobacteriosis. Int $\mathrm{J}$ Environ Res Public Health 2013;10:5886-5907.

[WHO] World Health Organization. World Health Organization Campylobacter Fact Sheets. 2019. Available at: https://www .who.int, accessed September 9, 2020.
Zhao S, Tyson GH, Chen Y, Li C, Mukherjee S, Young S, Lam C, Folster JP, Whichard JM, Mcdermott PF. Wholegenome sequencing analysis accurately predicts antimicrobial resistance phenotypes in Campylobacter spp. Appl Environ Microbiol 2016;82:459-466.

Address correspondence to:

Kun C. Liu, PhD

Applied Technology Center

at the Pacific Northwest Laboratory (PNL)

Office of Regulatory Science

Office of Regulatory Affairs (ORA)

The U.S. Food and Drug Administration

22201 23rd Drive SE

Bothell, WA 98021

USA

E-mail: kun.liu@fda.hhs.gov 